\title{
Éditorial
}

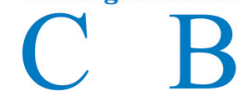

\section{Plagiat et autres mauvaises pratiques}

\author{
Jacky Samson*
}

N'importe quelle idée semble personnelle dès qu'on ne se rappelle plus à qui on l'a empruntée.

Jules Renard

Le plagiat peut être défini comme « l'acte de quelqu'un qui, dans le domaine artistique ou littéraire, donne pour sien ce qu'il a pris à l'œuvre d'un autre » (Larousse en ligne) mais il n'a pas d'existence légale. Le plagiat est assimilé à une contrefaçon. Selon l'article L.335-3 du Code de la Propriété Intellectuelle, la contrefaçon correspond à la « reproduction, représentation ou diffusion, par quelque moyen que ce soit, d'une œuvre de l'esprit en violation des droits de l'auteur ». Le plagiat représente donc le vol d'une création originale et toute contrefaçon des «œuvres de l'esprit » est passible de sanctions pénales.

Le plagiat a connu un premier essor avec l'invention de l'imprimerie mais, pendant longtemps, il fut plus considéré comme un emprunt, une source d'inspiration et beaucoup d'auteurs célèbres (Montaigne, La Fontaine, Molière, Voltaire, Rousseau...) y eurent recours sans que quiconque s'en offusque réellement. Certains moyens technologiques modernes (photocopieuse, internet) ont facilité le recours au plagiat. Depuis quelques années, le plagiat fait régulièrement la une des médias: tour à tour, des écrivains (Troyat, Minc, Attali, Deforges...), des journalistes (Poivre d'Arvor, Ardisson, MacéScaron...), des hommes politiques ou publics (Rama Yade, Bernheim...), des artistes (Khaled, Cologero, Luc Besson...), des universitaires (c'est sans aucun doute la liste la plus longue)... ont été suspectés, et le plus souvent condamnés pour plagiat. Toutefois, la distinction entre plagiat et inspiration n'est pas toujours facile à établir mais, le plus souvent, il s'agit d'un « emprunt servile » bien différent de l' « emprunt créatif » [1] que réalisaient les anciens. Certains n’hésitent pas à reproduire des passages entiers d'une œuvre, d'autres se livrent au pillage systématique de plusieurs auteurs (récemment, une thésarde de la Sorbonne semble avoir plagié plus de 40 auteurs pour son seul travail de thèse ! [2]). Certains ne succomberont qu'une fois, d'autres sont des récidivistes. Certains - peu nombreux - reconnaissent facilement leur forfait, d'autres - c'est-à-dire la plupart - s'évertuent à prouver leur bonne foi en usant de divers stratagèmes plus ou moins fallacieux (c'est une réminiscence, un plagiat réalisé par le documentaliste ou le nègre, une intertextualité...).

Le plagiaire qui trompe le lecteur sur l'origine de ses sources, s'approprie tout naturellement les éléments plagiés et les intègre dans sa propriété intellectuelle. Comme rédacteur en chef de la revue $M B C B$, j'ai reçu une demande de retrait d'un article quelques jours après sa parution en ligne, au motif que cet article plagiait la thèse du requérant. Après enquête, il s'est avéré que l'accusation de plagiat reposait uniquement sur une courte phrase que l'auteur de l'article et le dénonciateur avaient repris texto d'un document de l'AFSSAPS de 2007. Comme ce comportement était probablement révélateur d'un dysfonctionnement intellectuel, il fut demandé à l'auteur de l'article qui venait de réaliser une large revue de la littérature, d'analyser succinctement la thèse de l'accusateur. Après une simple lecture, il identifia plusieurs passages qui avaient été plagiés sur deux auteurs. Une autre fois, on me demanda de donner un avis sur une accusation de plagiat : un directeur de thèse était accusé par un de ses élèves d'avoir publié un article reprenant largement sa thèse sans le citer. Le plagiat était incontestable mais le plagié devint vite suspect lorsqu'on prit connaissance de sa thèse. Il fut assez facile de retrouver le document où il avait trouvé l'inspiration en recopiant in extenso de nombreux passages. Le plagiaire est le plus souvent très suspicieux car il ne peut pas s'imaginer que les autres soient différents de lui : il est donc convaincu qu'on va le plagier. De plus, il fait souvent preuve d'un certain aplomb : dernièrement, un article sur le plagiat [3] a du être rétracté... pour plagiat! Le plagiat semble devenir un véritable fléau dans le monde scientifique suscitant récemment l'organisation d'un congrès spécifique [4].

Le plagiat permet de suppléer un manque de créativité, de masquer une parésie de l'esprit, de rattraper le temps perdu,

\footnotetext{
*Correspondance : jacky.samson74@gmail.com
}

This is an Open Access article distributed under the terms of the Creative Commons Attribution License (http://creativecommons.org/licenses/by/4.0), which permits unrestricted use, distribution, and reproduction in any medium, provided the original work is properly cited 


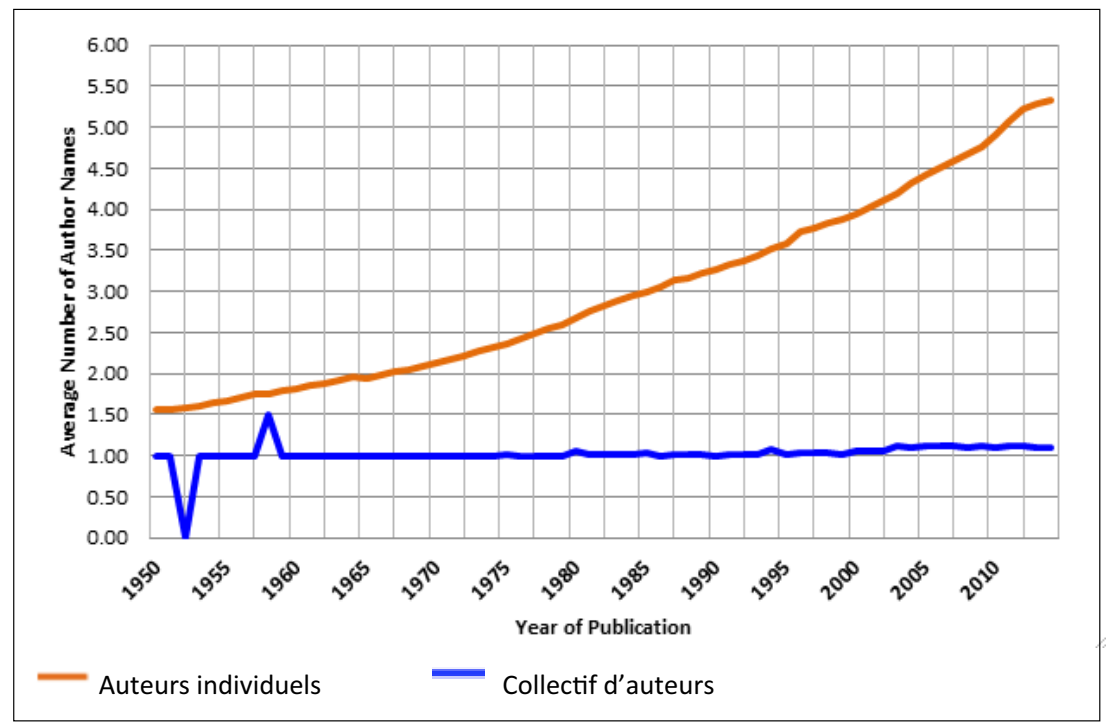

Fig. 1. Nombre moyen d'auteurs (individuel ou collectif) dans les publications selon les citations Medline / Pubmed. http : // www.nlm.nih.gov.bsd/autors1.html

de prolonger sa flemme... mais surtout d'atteindre un rythme de publications effréné, même pour ceux qui n'ont pas lu les " petits conseils » de Grégoire Chamayou [5] ou qui ne sont pas documentés sur la technique de publications mise au point à l'IPAG Business School de Paris [6]. Ainsi, en peu de temps, on peut donner du corps à son CV, augmenter son impact factor et son facteur H... et donc passer devant tous ces besogneux qui travaillent consciencieusement. Au plagiat, toujours dans le même but, on peut ajouter l'auto-plagiat (« salami factor »), la manipulation des résultats pour les rendre plus attractifs (« embellissement des résultats »), la création de données... Toujours dans le même but, on assiste à une inflation du nombre d'auteurs par article (fig. 1) : c'est un petit jeu ou une lutte sans merci pour certains afin d'apparaître comme auteur, sous divers prétextes, sans avoir réellement participé au travail. Par exemple, on peut se demander comment un collègue français (qui a reçu entre autres le 3 juin dernier le Grand Prix scientifique 2015 de la Fondation Louis D. - Institut de France), ayant en plus des activités annexes, peut être considéré comme l'auteur de 2054 publications : même pour un stakhanoviste, cela semble matériellement irréalisable si l'on respecte les critères de l'ICMJE (International Committee of Medical Journal Editors) pour bénéficier du titre d'auteur, à moins d'être dans une situation qui vous fait bénéficier en permanence d'une dilatation du temps. Cela signifie que le système dysfonctionne probablement à plusieurs niveaux, en particulier dans le comité de rédaction des revues. En réaction à un article qui venait de lui être refusé, William G. Hoover changea le titre et ajouta un troisième auteur (Stronzo Bestiale que l'on traduit le plus souvent par connard ou total asshole) avant de le soumettre à une autre revue qui le publia sans rien remarquer [7]. Hoover avait découvert l'expression stronzo bestiale lors d'un vol transatlantique qu'il avait effectué à côté de deux Italiennes qui ne cessaient d'employer ce terme dans leur conversation. Dès qu'il rencontra un collègue italien, il lui demanda la traduction : Hoover comprit alors qu'il avait trouvé le nom d'un nouveau collaborateur ! Il a eu le loisir de récidiver avant que la supercherie ne fut découverte et, presque 30 ans plus tard, l'article initial n'a toujours pas été rétracté (il est encore en vente pour $43,95 €)$ et Stronzo Bestiale, selon Scopus, serait actuellement affilié à l'Institut de physique expérimentale de Vienne (Autriche)!

Le plagiat constitue une réalité de plus en plus prégnante à l'Université mais il « est encore considéré comme une vue de l'esprit, un fantasme de collègues frustrés, un combat de chevaliers blancs aux intérêts suspects »[1]. On peut faire la même constatation pour la fraude qui représente la principale cause de rétractation des articles (fig. 2). Pour lutter contre le plagiat, des logiciels de détection ont été mis au point et sont à disposition dans certaines Universités et de plus en plus utilisés par les éditeurs mais il semble assez facile de ne pas se faire piéger par les algorithmes de détection. Au moins permettent-ils aux plagiaires de vérifier si leur mystification a réussi. Quant à l'auto-plagiat, il devrait être facilement détecté si les comités de rédaction et les éditeurs étaient tous vigilants. Par contre, la fraude est beaucoup plus difficile à détecter et elle est souvent découverte tardivement ou grâce au site Pubpeer où chacun peut, de façon anonyme, commenter un article ou dénoncer une fraude. C'est en grande partie grâce à ce site que les manipulations d'Olivier Voinnet [8] ont été révélées. Ironie de l'histoire, le créateur du site Pubpeer, 


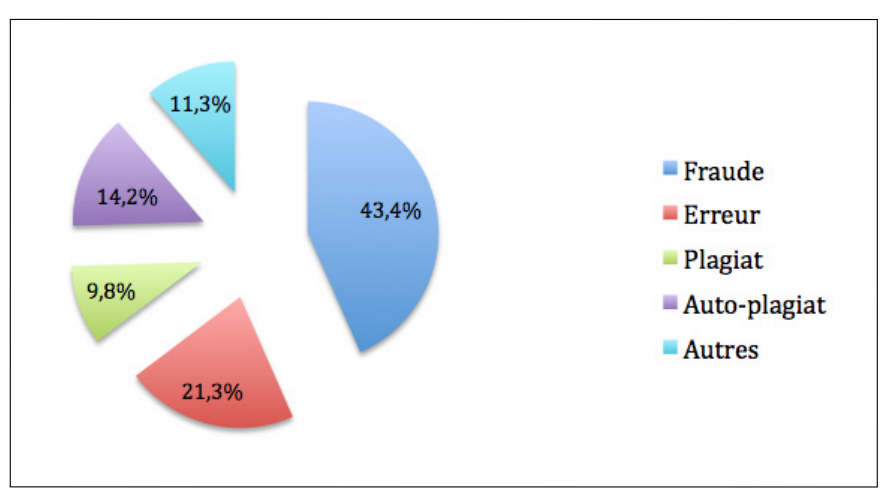

Fig. 2. La fraude biomédicale en chiffres : les causes de rétractations de 1977 à 2012. Pigenet Y, Ben Ytzhak L. Fraude : mais que fait la recherche ? CNRS Le Journal 2014;278:16-23.

actif depuis 2013, est un collègue de Voinnet. Depuis septembre 2015, on sait que c'est Brandon Steel, un américain en poste au CNRS depuis 2008. Le Ministère de l'Enseignement Supérieur et de la Recherche et le Comité d'éthique du CNRS n'ont pas attendu l'affaire Voinnet pour rédiger respectivement un rapport [9] et un guide [10] pour tenter de promouvoir l'intégrité scientifique. Le rapport expose sans détour la situation et la résume ainsi : « Ce qui manque le plus dans la situation française est : un traitement explicite et décomplexé de la fraude » et « une vue partagée... sur le traitement des plaintes ». Malheureusement, on attend toujours la mise en œuvre des recommandations de ce rapport.

Plagiat et fraude semblent en grande partie liés à la pression pour publier mais ce sont surtout ceux dont l'intégrité est malléable qui succombent les premiers. Dans un monde où l'on considère les indices bibliométriques suffisamment pertinents pour évaluer un individu, un service ou un laboratoire, certains sont tentés d'utiliser des artifices frauduleux. Tant que ces indices seront l'objet de vénération et/ou adulation et qu'il n'y aura pas de sévères sanctions prises envers les fraudeurs, la fraude et le plagiat continueront de gangréner la littérature scientifique. Certains, comme Alain Fuchs, président du CNRS, l'ont bien compris. Il donne un avis très nuancé sur ces indices: «l'usage inapproprié et systématique d'indicateurs chiffrés (facteurs d'impact, facteur « $h$ », etc.) à tendance à fausser le jeu. La qualité d'un travail scientifique s'évalue par les pairs, elle ne se mesure pas par des chiffres. Je tiens d'ailleurs à signaler au passage que le Comité scientifique résiste autant qu'il le peut à cette furie des indicateurs» [11].
Cet entretien a eu lieu après la condamnation d'Olivier Voinnet ; il est fort probable qu'auparavant Alain Fuchs avait un avis moins négatif sur les indices bibliométriques. Le système risque encore de perdurer car les autorités politiques et universitaires pratiquent le plus souvent l'amphibologie et/ou la procrastination. En attendant cette évolution, si on n'a pas le pessimisme de Jean-François Revel (cf. infra), peut-être pourrait-on prendre de réelles sanctions (d'un niveau comparable à la condamnation infligée dernièrement à Dong-Pyou Han aux USA : 4 ans et 9 mois de prison et 7,2 millions \$ pour fraude scientifique) car les plagiaires et les fraudeurs ont le plus souvent la résipiscence fugace.

L'homme n'a pas besoin qu'on le force à être intellectuellement malhonnête pour le devenir. Il y parvient très bien tout seul. Jean-François Revel

\section{Références}

1. Maurel-Indart H. Petite enquête sur le plagiaire sans scrupule. Editions Léo Scheer, Paris, 2013.

2. Darde JN. L'Université Paris-Sorbonne (Paris IV) recrute une plagiaire. http://archeologie-copier-coller.com

3. Shamin T. Development of a guideline to approach plagiarism in Indian senario. Indian J Dermatol 2014;59:473-5.

4. $\mathrm{II}^{\text {nd }}$ International conference plagiarism across Europe and beyond 2015 (10-12 juin 2015).

5. Chamayou G. Petits conseils aux enseignants-chercheurs qui voudront réussir leur évaluation. http://www.contretemps.eu/ interventions/

6. Gless E. IPAG : les secrets d'une progression « fulgurante » en recherche. http://letudiant.fr/educpros/enquetes

7. Moran B, Hoover WG, Bestiale S. Diffusion in a periodic Lorentz gas. J Stat Phys 1987;48:709-26.

8. Morin H, Larousserie D. Olivier Voinnet, star de la biologie végétale, sanctionné par le CNRS. http://www.lemonde.fr/ sciences/article2015/07/10

9. Alix J-P. Renforcer l'intégrité dans la recherche en France. Propositions de prévention et de traitement de la fraude. Ministère de l'Enseignement Supérieur et de la Recherche (septembre 2010).

10. Comité d'éthique du CNRS. Promouvoir une recherche intègre et responsable (juillet 2014).

11. Entretien d'Alain Fuchs avec Louise Lis. Ne transigeons pas avec l'intégrité. CNRS Le Journal 2015;281:56-7.

PS : Cet éditorial tombe fort à propos pour vous informer, « adoré » lecteur que MBCB aussi est victime de ce genre de pratique et que des plagiats avérés ont été interceptés très récemment. Nous, Rédacteurs en Chef et Editeur avons pris la décision de passer désormais toutes les soumissions à MBCB au détecteur de copier-coller (plus communément appelé détecteur anti-plagiat). Nous sommes conscients des limites de l'outil, fort justement rappelées dans l'éditorial ci-dessus. Au-delà de $20 \%$ d'emprunts, les auteurs sont informés et leur soumission immédiatement interrompue. Ce système fonctionne très bien !

Jean-Christophe Fricain et Philippe Lesclous 